\title{
MYCN drives glutaminolysis in neuroblastoma and confers sensitivity to an ROS augmenting agent
}

Tingting Wang', Lingling Liu', Xuyong Chen', Yuqing Shen $\mathbb{1}^{1}$, Gaojian Lian', Nilay Shah', Andrew M Davidoff², Jun Yang $\mathbb{B}^{2}$ and Ruoning Wang (i)

\begin{abstract}
Heightened aerobic glycolysis and glutaminolysis are characteristic metabolic phenotypes in cancer cells. Neuroblastoma (NBL), a devastating pediatric cancer, is featured by frequent genomic amplification of MYCN, a member of the Myc oncogene family that is primarily expressed in the early stage of embryonic development and required for neural crest development. Here we report that an enriched glutaminolysis gene signature is associated with MYCN amplification in children with NBL. The partial knockdown of MYCN suppresses glutaminolysis in NBL cells. Conversely, forced overexpression of MYCN in neural crest progenitor cells enhances glutaminolysis. Importantly, glutaminolysis induces oxidative stress by producing reactive oxygen species (ROS), rendering NBL cells sensitive to ROS augmentation. Through a small-scale metabolic-modulator screening, we have found that dimethyl fumarate (DMF), a Food and Drug Administration-approved drug for multiple sclerosis, suppresses NBL cell proliferation in vitro and tumor growth in vivo. DMF suppresses NBL cell proliferation through inducing ROS and subsequently suppressing MYCN expression, which is rescued by an ROS scavenger. Our findings suggest that the metabolic modulation and ROS augmentation could be used as novel strategies in treating NBL and other MYC-driven cancers.
\end{abstract}

\section{Introduction}

Heightened aerobic glycolysis (i.e., the "Warburg effect") and glutaminolysis are characteristic hallmarks of cancer cells $^{1-5}$. Both processes are tightly controlled to fulfill cell growth-associated and proliferation-associated bioenergetics, biosynthetic, and redox demands. While tissue microenvironments play a role in homeostatic regulation of cell metabolism, the metabolic rewiring of cancer cells is largely driven by a hierarchical oncogenic cascade involved in Akt/mTOR, mitogen-activated protein kinase signaling, and a hypoxia-inducible factor 1 (HIF1)-

Correspondence: Jun Yang (Jun.Yang2@STJUDE.ORG) or Ruoning Wang (ruoning.wang@nationwidechildrens.org)

${ }^{1}$ Center for Childhood Cancer and Blood Diseases, Hematology/Oncology and BMT, The Research Institute at Nationwide Children's Hospital, The Ohio State University, Columbus, OH, USA

${ }^{2}$ Department of Surgery, St. Jude Children's Research Hospital, Memphis, TN 38105 USA

Edited by G Raschellà. dependent and Myc-dependent metabolic transcriptome ${ }^{4,6}$. By analogy to the concept of oncogene addiction $^{7}$, we envision that a persistent metabolic rewiring renders cancer cells highly dependent on certain metabolic pathways in a way that other cells are not (metabolic addiction), hence modulation of this process holds the promise of novel metabolic interventions (metabolic vulnerability).

Neuroblastoma (NBL) is an embryonal malignancy of early childhood, arising from sympathoadrenal precursors that have evaded terminal differentiation and proliferated uncontrollably. Approximately half of the patients with NBL are considered "high risk," as defined by clinical, radiographic, and biological criteria. These patients have a high rate of treatment failure, most commonly due to disease progression early in treatment or relapse at the end of multimodal therapy. These failures make NBL the deadliest extracranial pediatric solid tumor, accounting 
for $15 \%$ of childhood cancer deaths ${ }^{8,9}$. Children with highrisk NBL are treated with aggressive multimodal therapy. Nevertheless, $<50 \%$ of patients with high-risk NBL will survive long term with current therapies, and survivors are at risk for serious treatment-related late toxicities. Therefore, novel treatments must be developed to enhance therapy efficacy with minimal toxicity, prevent disease recurrence, and maintain durable cures.

While several genetic abnormalities (ALK, PHOX2B, Let-7, ATRX, PTPN11, etc.) are known to contribute to the pathogenesis of subsets of NBL, genomic amplification of the Myc oncogene family member, MYCN, occurs in about $50 \%$ of high-risk NBL cases and is the most prevalent genetic abnormality identified in $\mathrm{NBL}^{10}$. MYCN is a potent oncogenic driver and the single worst prognostic biomarker in NBL, with MYCN amplification indicating $<30 \%$ chance of survival ${ }^{11}$. It has been suggested that MYCN regulates the transcription of some metabolic enzymes and transporters involved in MYCNamplified NBL cell lines ${ }^{12,13}$. Also, activating transcription factor 4 (ATF4) and HIF1 are involved in regulating the transcription of metabolic genes in glutamine and glucose metabolic pathways, respectively ${ }^{12,14,15}$. The concept of metabolic reprogramming and its role in cell fate determination is well established in metabolic diseases, and, more recently, it has been applied to many adult cancers $^{3,16,17}$. However, the impact of metabolic reprogramming of cancer cells by oncogenes is not entirely clear. How to harness the impact of metabolic reprogramming to develop novel therapies is also very important for cancer treatment. A better understanding of how genetic alterations (MYCN amplification) impact NBL metabolic reprogramming will enable us to identify key oncogenic events and metabolic characters, and to devise effective therapies.

Here, we report a role of MYCN in regulating NBL metabolic reprogramming and reactive oxygen species (ROS) induction. The short hairpin RNA (shRNA)-mediated partial knockdown of MYCN suppresses the expression of metabolic genes and the activity of glutaminolysis in NBL cell lines. Heightened glutaminolysis in NBL cells by MYCN provides bioenergetic support and induces ROS as a by-product in mitochondria, conferring metabolic vulnerability of NBL cells to ROSproducing agent as cancer cells are more sensitive, than normal cells, to agents that cause further accumulation of ROS. We identified dimethyl fumarate (DMF), a Food and Drug Administration (FDA)-approved drug for inflammation and autoimmunity, as a novel therapeutic agent that suppresses NBL cell growth through inducing ROS and subsequently suppressing MYCN expression. Our studies suggest that metabolic modulation of glutaminolysis and ROS augmentation may represent effective strategies in treating NBL and other MYC-driven cancers.

\section{Results \\ MYCN is required for driving glutaminolysis in MYCN- amplified NBL}

Although it has been well recognized that the protooncogene, MYC, is responsible for orchestrating a transcriptional program driving metabolic reprogramming in many adult tumors, the role of MYC, specifically its family member MYCN, during metabolic reprogramming in pediatric cancers including NBL is not fully understood. We, therefore, sought to determine whether MYCN is involved in regulating metabolic programs in MYCNoverexpressed NBL. By cross-referencing the published microarray and genetic data, we found that an enriched glutaminolysis gene signature is associated with MYCN amplification in children with NBL (Fig. 1a). Next, we sought to assess whether the genetic modulation of MYCN impacts NBL cell metabolism. The transient knockdown of MYCN by small interfering RNA (siRNA) did not allow us to obtain sufficient amount of cells required for metabolic assays. Therefore, we established NB-1643 (a NBL cell line with MYCN overexpression) stably expressing a doxycycline (Dox)-inducible MYCNshRNA, which not only allows us to acutely knockdown endogenous MYCN level following Dox treatment, but also allows us to obtain sufficient amount of cells to examine metabolic activity following MYCN knockdown. Immunoblot (IB) and quantitative PCR (qPCR) analysis confirmed the modest downregulation of MYCN (Fig. 1b). Then, we utilized radiochemical-based approaches to assess the metabolic activities in control and MYCNshRNA knockdown groups. The results showed that even modest downregulation of MYCN led to a $50 \%$ reduction in glutaminolysis rate compared to the control group, as indicated by ${ }^{14} \mathrm{CO}_{2}$ release from $\left[\mathrm{U}_{-}{ }^{14} \mathrm{C}\right]$ glutamine (Fig. 1c). Meanwhile, the rate of glycolysis, measured by the detritiation of $\left[5-{ }^{3} \mathrm{H}\right]$ glucose, did not differ significantly between the two groups, suggesting that glutaminolysis is more sensitive to the loss of MYCN than glycolysis. Consistent with a reduced glutaminolysis activity, the expression of metabolic genes involved in glutaminolysis is dampened to different extents following acute induction of MYCN-shRNA knockdown (Fig. 1d and Fig. S1A). These results were supported by a different shRNA knockdown of MYCN in NB-1643 cells (Fig. S1C, S1D), which resulted in suppression of glutaminolysis but not glycolysis (Fig. S1C), despite that the second shRNA had a lower knockdown efficiency. To further crossvalidate that $\mathrm{MYCN}$ is responsible for regulating glutaminolysis, we took additional step-wise approaches. We first transfected NB-EBC1 (MYCN-overexpressed NBL cell line) with MYCN siRNA, and confirmed that siRNAmediated knockdown of MYCN in NB-EBC1 also resulted in the downregulation of metabolic genes involved in glutaminolysis, albeit with some discrepancies on genes 


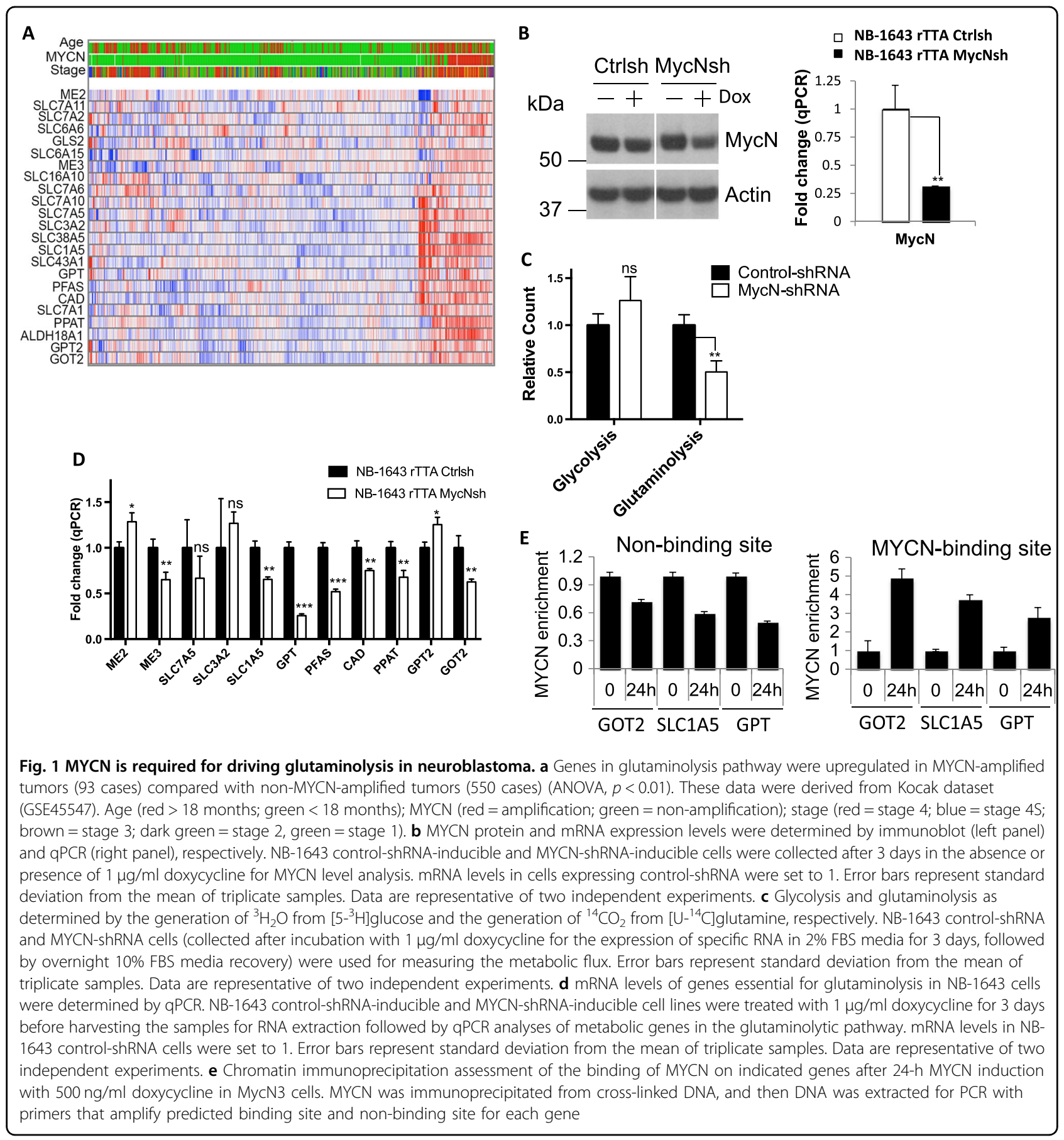

including ME2, SLC7A5, and SLC3A2, compared to the result in NB-1643 (Fig. S1B and Fig. 1d). We reasoned that the difference in cell lines (NB-1643 vs. NB-EBC1) and knockdown methods (shRNA vs. siRNA) likely contributed to these discrepancies. Next, we used a gain-offunction approach to further strengthen the conclusion that MYCN plays a role in regulating glutaminolysis. We first employed a recently established cellular model based on the genetic manipulation and transformation of the
JoMa cells, a murine multipotent neural crest progenitor cell line ${ }^{18,19}$. JoMa cells can be kept in an undifferentiated state, differentiated into a variety of cell lineages, or transformed into NBL cells by MYCN overexpression in vitro and developed to NBL after transplantation in vivo ${ }^{18,19}$. This model allowed us to analyze the molecular and metabolic alterations in NBL progenitor cells. As such, we generated a lentiviral construct to overexpress MYCN to mimic MYCN amplification in NBL. 
Our results showed that the overexpression of MYCN in JoMa cells promotes glutaminolysis, but not glycolysis in these cells (Fig. S1E). Then, we used a Dox-inducible MYCN cell line $(\mathrm{MycN} 3)^{20}$ and further showed that acute induction of MYCN significantly elevated glutaminolysis but not glycolysis in NBL cells (Fig. S1F). Finally, we sought to examine whether MYCN directly controls the transcription of the metabolic genes involved in glutaminolysis. Again, we employed the MycN3 cell line that enabled us to acutely induce the expression of MYCN, thereby assessing the binding of MYCN on the predicated binding or non-binding site (intrinsic negative control) in each candidate gene by chromatin immunoprecipitation and polymerase chain reaction (ChIP-PCR). After $24 \mathrm{~h}$ induction, MYCN binding was greatly enriched in the promoter regions but not negative control sites (predicated non-binding sites) of the genes including GOT2, $S L C 1 A 5$, and GPT that we randomly chose to examine (Fig. 1e). Together, these results suggest that MYCN plays a critical role in the regulation of glutaminolysis in NBL.

\section{Glutaminolysis increases the production of ROS and renders NBL cells sensitive to oxidative stress}

Previous studies demonstrated that glutamine is an essential metabolic substrate driving cancer cell growth through fueling mitochondrial oxidation and providing carbon and nitrogen for the biosynthesis of other macromolecules ${ }^{2}$. Consistent with these studies, our results showed that the removal of glutamine from culture medium significantly dampened the growth of MYCNoverexpressed NBL cells. However, the supplement of dimethyl $\alpha$-ketoglutarate (DMKG, cell-permeable analog of $\alpha-\mathrm{KG})$, a downstream metabolite of glutamine, restored cell growth in the absence of glutamine (Fig. 2a and Fig. S2A). These results suggest that glutaminolysis supports NBL cell growth by providing $\alpha-K G$, an anaplerotic substrate of the tricarboxylic acid (TCA) cycle, fueling oxidative phosphorylation in mitochondria. It is known that enhanced oxidative phosphorylation through mitochondrial electron transport chain (ETC) produces ROS as a by-product, which is required for promoting cell proliferation and transformation but also leaves cells more vulnerable to further oxidative stress ${ }^{21,22}$. Therefore, we further investigated whether MYCN-dependent glutaminolysis impacted ROS production and cellular response to oxidative stress. Our results showed that overexpression of MYCN in JoMa cells increased the fluorescent intensity of $\mathrm{H}_{2}$ DCFDA (DCF) (Fig. 2b), indicating elevated levels of intracellular hydrogen peroxide $\left(\mathrm{H}_{2} \mathrm{O}_{2}\right)$ that is the end product following ROS disputation. As illustrated in Fig. 2c, the addition of $\mathrm{H}_{2} \mathrm{O}_{2}$ suppressed the proliferation of MYCN-transformed JoMa cells but not control JoMa cells. Consistent with this, $\mathrm{H}_{2} \mathrm{O}_{2}$ treatment significantly suppressed the proliferation of $\mathrm{MYCN}$-overexpressing
NBL cell lines (Fig. 2d and Fig. S2B). We further discovered that the removal of glutamine from culture media (glutamine starvation) or the addition of glutaminolysis inhibitor 6-diazo-5-oxo-L-norleucin (DON) significantly reduced ROS levels in MYCN-overexpressing JoMa cells (Fig. S2C). Together, our data suggest that MYCN-driven glutaminolysis increases ROS generation and sensitizes NBL cells to oxidative stress.

\section{Identification of DMF that suppresses NBL cell proliferation}

Next, we selected a list of compounds with activities against a range of cell catabolic pathways and examined their impact on NBL cell growth (Supplemental Table 1). While some compounds suppressed the growth of MYCN-overexpressed NBL cell lines (Fig. S3A), we chose to follow-up with DMF, which exhibited strong inhibition of NBL cells growth and was also suggested to induce ROS through binding and depleting intracellular glutathione $(\mathrm{GSH})^{23,24}$. Our gene enrichment analysis revealed an enriched oxidative response gene signature in DMF-treated NBL cells (Fig. S3B), confirming that DMF treatment induces oxidative stress. DMF is the key active ingredient of BG-12/TECFIDERA and FUMADERM; both have been approved for treatment against the autoimmune diseases multiple sclerosis (MS) and psoriasis $^{23,24}$. As previous studies showed that DMF exhibited anti-inflammatory activities against immune cells with doses ranging from 20 to $100 \mu \mathrm{M}^{25,26}$, we applied a similar dose range to NBL cells and observed a dose-dependent suppression of cell growth and induction of cell death (Fig. 3a, b and Fig. S3C). Given that DMF is a cellpermeable form of fumarate, a TCA cycle metabolite, we then investigated whether other TCA cycle metabolites displayed similar anti-tumor activity as DMF does. DMKG, a cell-permeable form of $\alpha$-ketoglutarate, failed to suppress NBL cell growth (Fig. 3c and Fig. S3D), suggesting the anti-tumor property is unique in DMF but not in other TCA cycle metabolites. As fumarase (FH) converts fumarate to malate during the TCA cycle, we hypothesized that the knockdown of FH increases fumarate and consequentially suppresses NBL cell growth. To test this hypothesis, we transiently transfected NBL cells with siRNAs targeting FH. As shown in Fig. S3E, FH siRNAs partially reduced the expression of $\mathrm{FH}$ in NBL cells and delayed NBL cell growth. Next, we sought to determine whether DMF had any anti-tumor activities in vivo. Given the known autoimmune-suppressive effect of DMF, we reasoned that testing DMF in an immunedeficient host might potentially exaggerate its anti-tumor effect. Therefore, we assessed the tumor-suppressive efficacy of DMF in a syngeneic NBL xenograft mouse model, which is based on a mouse NBL cell line derived from TH-MYCN transgenic mice, allowing us to establish NBL xenograft in immunocompetent hosts ${ }^{27}$. Our data 


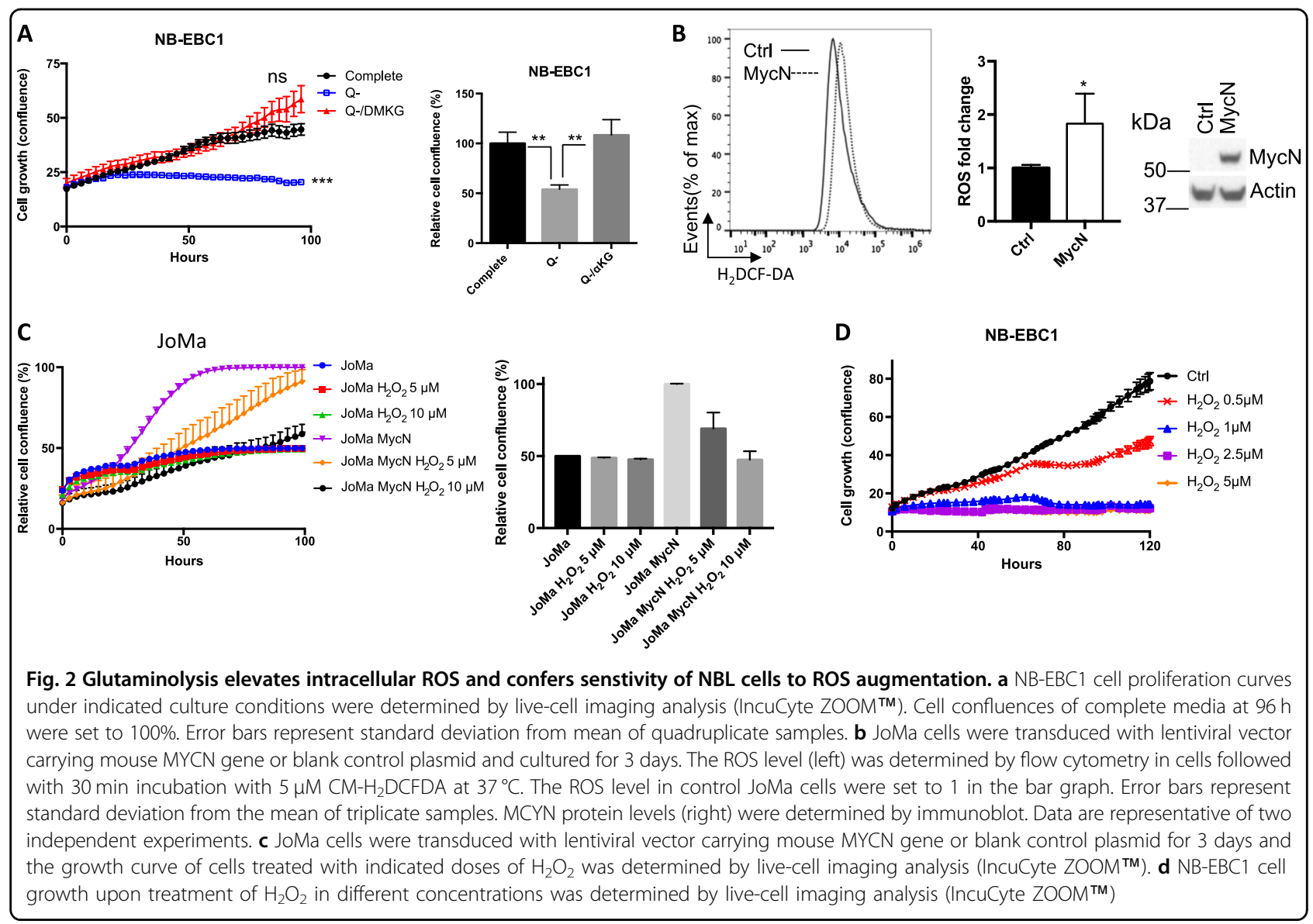

showed that DMF treatment significantly delayed NBL tumor growth in this model (Fig. 3d, e). In addition to its known immune-suppressive activities, our data have shown that DMF exhibited the anti-tumor activity in MYCN-overexpressing NBL cell lines and in mouse NBL xenografts.

\section{DMF suppresses the expression of MYCN through augmenting ROS}

To gain more mechanistic insights on the effects of DMF on NBL cells, we analyzed our gene expression data and found that a number of Myc target gene signatures were significantly suppressed following DMF treatment (Fig. 4a). Our results showed that the transient knockdown of MYCN suppressed NBL proliferation, confirming the role of MYCN as a key oncogenic driver in these cell lines (Fig. S4A, S4B). Next, we examined the level of MYCN in NBL cell lines following DMF treatment. As Fig. 4b, c showed, DMF suppresses the expression of MYCN at both the mRNA and the protein levels. To further explore the molecular mechanisms behind the suppression of MYCN expression and NBL growth upon DMF treatment, we assessed the levels of ROS following DMF treatment. Consistent with the induction of oxidative response genes (Fig. S3B), DMF treatment increased the intracellular ROS levels in NBL cells (Fig. 4d). We then treated NBL cells with $\mathrm{H}_{2} \mathrm{O}_{2}$ to mimic the accumulation of intracellular ROS. Consistent with the effect on cell growth (Fig. $2 \mathrm{~d}$ and Fig. S2B), $\mathrm{H}_{2} \mathrm{O}_{2}$ treatment reduced the levels of $\mathrm{MYCN}$ in a dosedependent manner (Fig. S4C), indicating that increased ROS suppressed MYCN expression. To determine whether ROS mediates the effects of DMF on NBL cells, we concurrently treated NBL cells with DMF and $N$ acetyl-L-cysteine (NAC), an ROS scavenger. As Fig. 4e, f showed, NAC restored the level of MYCN and the proliferation of NBL cells following DMF treatment. In addition, GSH reduced ethyl ester (GSH-MEE), a membrane-permeable form of antioxidant, also protected NBL cells against the DMF-mediated anti-proliferation effect (Fig. S4D). Finally, we sought to examine whether genetic knockdown of FH suppresses NBL cell proliferation through ROS induction. The addition of NAC had no impact on cell growth in control group; however, it partially restored cell growth in the FH siRNA group (Fig. S4E). Collectively, our results suggest that DMF suppresses MYCN expression and cell growth through augmenting the level of ROS. 


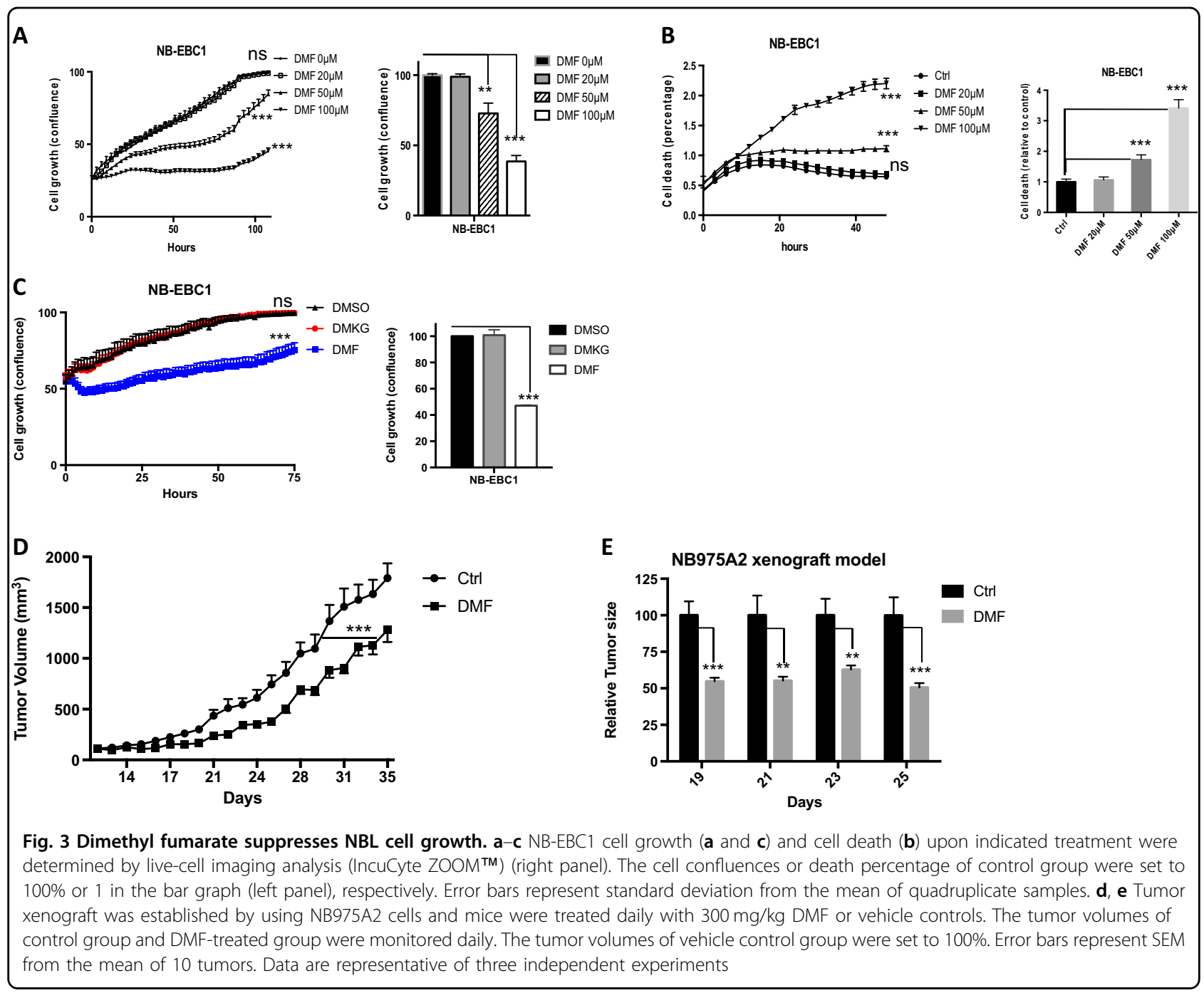

\section{DMF induces a compensatory NRF2-mediated response}

We have shown the induction of an enriched oxidative response gene signature in NBL cells following DMF treatment (Fig. S3B). Consistent with previous studies in other cells $^{23,24}$, we found DMF treatment directly induced ROS levels in NBL cells (Fig. 4d). A central transcriptional mechanism of defending against oxidative stress is through the activation of nuclear factor erythroid 2related factor 2 (NRF2), which drives the expression of a wide range of metabolic genes involved in producing, regenerating, and utilizing GSH. NRF2 is also involved in modulating thioredoxin (TXN), NADPH generation, and iron sequestration. The expression of these genes therefore converges on suppressing oxidative stress ${ }^{28}$. Previous studies suggested that DMF could covalently modify Kelch-like ECH-associated protein 1 and stabilize NRF2 ${ }^{29}$. Consistent with this, DMF treatment significantly induced the expression of NRF2 at the protein level (Fig. 5a). In addition, the transcription of four canonical NRF2 targets was induced in NBL cells following DMF treatment in a dose-dependent and time-dependent manner (Fig. 5b). The siRNA-mediated knockdown of NRF2 partially suppressed the induction of three canonical NRF2 targets, CD44, Txnrd, and XCT upon DMF treatment (Fig. S5A). Nevertheless, the combination of NRF2 siRNA and DMF treatment led to a significantly higher level of ROS compared to DMF treatment alone (Fig. 5d). These results suggest that NRF2-mediated anti-oxidative response may protect cells from DMF treatment. Since DMF treatment suppresses NBL cell growth and MYCN expression in a ROS-dependent manner (Fig. 4e, f), we next assessed MYCN level and cell growth following the treatment of DMF, NRF2 siRNA or the combination of DMF and NRF2 siRNA. Our data showed that the combination of DMF and NRF2 siRNA resulted in the greatest suppression on MYCN levels (Fig S5B) and NBL cell growth (Fig. 5c). Taken together, our data suggest that DMF treatment readily induces a robust NRF2-mediated anti- 


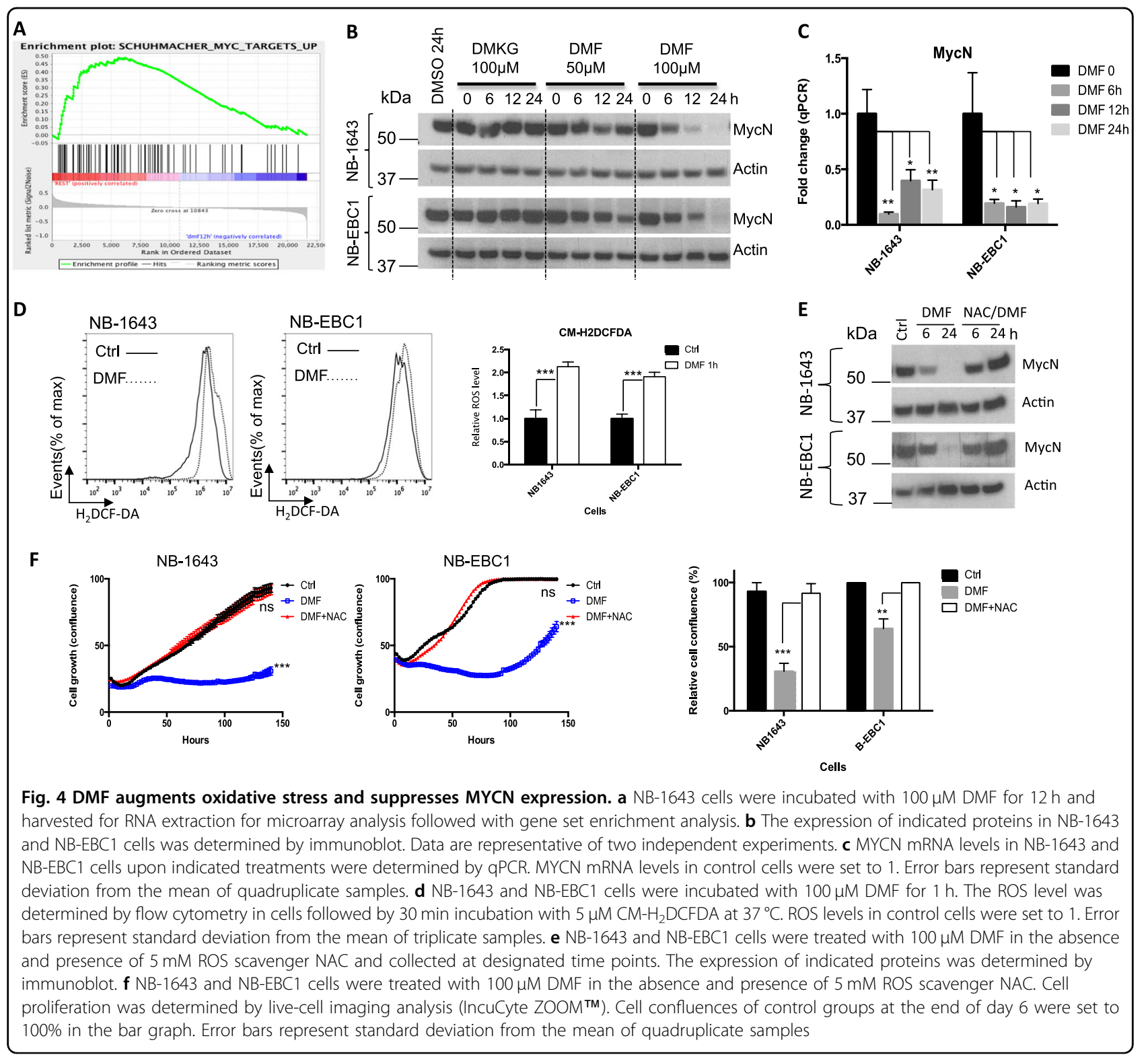

oxidative response, which might play a compensatory role in defending against ROS induction following DMF treatment.

\section{Discussion}

NBL like many other pediatric cancers arises from embryonal cell hyperplasia and therefore possesses embryonal features including the metabolic state associated with embryonic development ${ }^{30}$. A better understanding of the regulatory mechanism and the metabolic state of pediatric cancer is essential for identifying potential therapeutic targets that are unique for tumor but not for normal tissues in a developmental stage. Protooncogene Myc is required for metabolic reprogramming in most adult cancers and in immune cells ${ }^{31-33}$. The dysregulation of MYCN, a member of the Myc family of oncogenes that is primarily expressed in the early stage of embryonic development and required for neural crest development, is a driver of many high-risk $\mathrm{NBL}^{30,34,35}$. In the current study, we have revealed a tight association between MYCN overexpression and the transcriptional upregulation of metabolic genes in glutaminolysis. Interestingly, the partial knockdown of MYCN suppresses glutaminolysis, but does not affect glycolysis.

Conventional chemotherapies target biological processes involved in cell proliferation, including cell division and DNA replication, which can cause significant toxicity to normal tissues. Chemotherapeutics, particularly those that cause DNA damage, can also induce malignant transformation, leading to secondary cancers. Thus, novel 


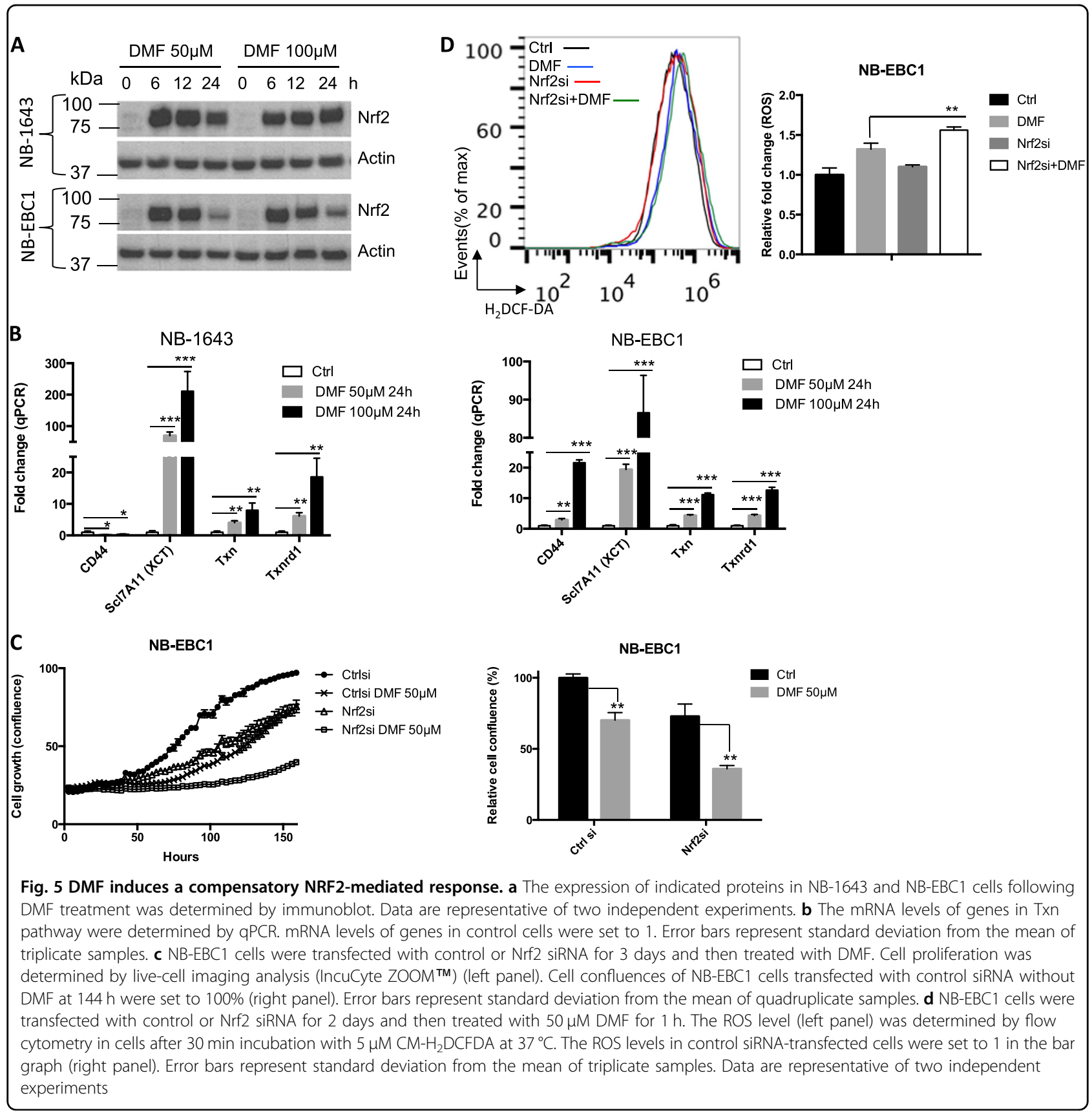

therapeutic targets are being identified that are more specific to cancer cell biology. One such approach is to target cancer metabolism ${ }^{4,31,36,37}$. The metabolic processes are hallmarks of cancers ${ }^{1,2}$, and therapeutic inhibition of these processes inherently have fewer systemic toxicities $^{38}$. Genomic amplification of the Myc oncogene family member, MYCN, occurs in about $50 \%$ of high-risk NBL and is the most prevalent genetic abnormality identified in NBL. MYCN is a potent oncogenic driver and the single worst prognostic biomarker in NBL, associated with $<30 \%$ chance of survival ${ }^{11}$. Recent studies suggest that pharmacological targeting BRD4, a member of the BET bromodomain family, can indirectly suppress MYC expression in various cancer models. However, the direct pharmacological targeting MYCN, which functions as a transcription factor, remains a challenge ${ }^{39}$. As a key MYCN-regulated process, the metabolic program is a critical biological pathway in cancer and has been recently validated as a therapeutic target ${ }^{38,40}$. Previous work demonstrated that NBL tumors overexpressed ornithine 
decarboxylase (ODC), a key metabolic enzyme in polyamine biosynthetic pathway, and that proliferation of preclinical models of NBL were suppressed by ODC inhibitor $\alpha$-difluoromethylornithine (DFMO) ${ }^{41}$. DFMO is currently used in two ongoing clinical trials to prevent or treat refractory and relapsed NBL, demonstrating the potential of targeting metabolic pathways ${ }^{41-44}$.

Cancer cells often display elevated levels of ROS compared with non-transformed cells. ROS are largely generated as by-products of the mitochondrial ETC, and is intimately involved in tumor initiation, tumor progression, and responses to therapy ${ }^{21,22}$. While low levels of ROS are involved in modulating oncogenic signaling pathways, promoting cancer tumor proliferation and survival, excessive ROS induces oxidative stress, which often renders tumors vulnerable to further stresses. Hence, strategic enhancement of oxidative stress either by augmenting ROS production or suppressing antioxidant capacity may kill cancer cells while sparing nontransformed cells ${ }^{37,45-47}$. We have shown that NBL cells are highly addicted to MYCN-driven glutaminolysis, which drives cell growth by providing bioenergetic support but also induces oxidative stress by producing ROS. Transient activation of MYCN enhances ROS production, indicating that MYCN-dependent glutaminolysis induces oxidative stress by producing ROS as a by-product. As such, we envision that the MYCN-dependent metabolic reprogramming and oxidative stress in NBL represents a critical metabolic vulnerability that could be exploited therapeutically by ROS augmenting agents. DMF treatment engaged a very rapid oxidative stress response in NBL cells, due to the augmentation of ROS production. ROS scavenger, NAC, annuls DMF-dependent suppression on cell growth. It has also been reported that DMF impacts the epigenetic machinery and nuclear factor- $\mathrm{KB}$ signaling in transformed cells ${ }^{48,49}$. DMF is a longapproved treatment for psoriasis in Germany ${ }^{50,51}$ and is approved by the FDA as a first-line oral treatment for relapsing MS (marketed as Tecfidera by Biogen) ${ }^{52,53}$. This long record of accomplishment in clinical use demonstrates that DMF is a safe drug with generally mild toxicities and may represent an optimal drug for future development.

\section{Materials and methods Reagents}

Cell culture materials and Matrigel were purchased from Fisher Scientific Inc. (Waltham, MA, USA). Glucose-free and glutamine-free Dulbecco's modified Eagle's media, Dox, DMKG, DON, $N$-acetyl-L-cysteine, and GSH reduced ethyl ester were purchased from SigmaAldrich Inc. (Saint Louis, MO, USA). DMF was from ACROS Organics (Geel, Belgium). Chicken embryo extract was from Gemini Biological Products (West
Sacramento, CA, USA). B-27 supplement and N-2 supplement were obtained from Gibco (Waltham, MA, USA). Anti-MYCN, anti-fumarate hydratase, anti-Nrf2, and anti-actin antibodies were from Santa Cruz Biotechnology (Santa Cruz, CA, USA).

\section{Cell culture}

Cell line identities have been authenticated by short tandem repeat analysis. NB-1643 and NB-EBC1 cells were kindly provided by Dr. Peter Houghton. NB975A2, a mouse NBL cell line derived from spontaneous tumor of TH-MYCN mice, was a gift from Dr. Rimas Orentas. All NBL cells used were grown in Dulbecco's modified Eagle's medium with $10 \%$ fetal calf serum in a $37^{\circ} \mathrm{C}$ humidified atmosphere of $95 \%$ air and $5 \% \mathrm{CO}_{2}$. MycN3 cells (a gift of Jason Shohet, Houston, TX, USA) were grown in RPMI1640 medium with $10 \%$ fetal calf serum in a $37^{\circ} \mathrm{C}$ humidified atmosphere of $95 \%$ air and $5 \% \mathrm{CO}_{2}{ }^{20}$. JoMa cells were grown on cell culture flask/dish coated with fibronectin, in NCC-medium supplemented with 10\% chicken embryo extract, B-27 supplement, N-2 supplement, and $200 \mathrm{~nm} 4-\mathrm{OHT}$, and the medium was changed daily $^{18,19}$.

\section{siRNA transfection}

The siRNA oligonucleotides corresponding to human MYCN, FH, and Nrf2 were purchased from Fisher Scientific Inc. siRNA oligonucleotides $(20 \mathrm{nM})$ were transfected into cells using Lipofectamine RNAiMAX reagent (Invitrogen). After $48-72 \mathrm{~h}$ of transfection, IBs were carried out to examine the knockdown of targeted proteins.

\section{Lentiviral transduction}

Plasmids encoding MYCN shRNA or mouse MYCN were purchased from Transomic Technologies (Huntsville, AL, USA). Lentiviruses were produced with the helper vectors in HEK293FT cells kindly provided by Dr. Peter Hunghton according to the protocol ${ }^{54}$. Lentiviruses, encoding for inducible MYCN-shRNA expression or mouse MYCN expression, were directly added to the cell culture medium and incubated for $48-72 \mathrm{~h}$ before further analysis.

\section{RNA isolation, reverse transcription, and qPCR}

Total RNA was isolated using the RNA extraction kit (Zymo and Qiagen) and was reverse transcribed using random hexamer and M-MLV Reverse Transcriptase (Invitrogen). SYBR green-based quantitative RT-PCR for specific genes was performed using the Applied Biosystems Real-Time PCR System. Samples for each experimental condition were run in triplicate and were normalized to $\beta$-2-microglobulin to determine relative expression levels. Primer sequences were obtained from PrimerBank $^{55}$. Primer sequences are listed in Table S2. 


\section{Protein extraction and western blot analysis}

Cells were harvested, lysed, and sonicated at $4{ }^{\circ} \mathrm{C}$ in a lysis buffer (50 mM Tris- $\mathrm{HCl}, \mathrm{pH} 7.4,150 \mathrm{mM} \mathrm{NaCl}, 0.5 \%$ sodium dodecyl sulfate, $5 \mathrm{mM}$ sodium pyrophosphate, protease and phosphatase inhibitor tablet). Cell lysates were centrifuged at $13,000 \times g$ for $15 \mathrm{~min}$, and the supernatant were recovered. The protein concentrations were determined by using the Pierce ${ }^{\mathrm{TM}}$ BCA Protein Assay kit (Thermo Fisher Scientific). After 5 min boiling in $4 \times$ NuPAGE $^{\circ}$ LDS Sample Buffer with $10 \times$ Reducing solution (Thermo Fisher Scientific), the proteins were separated by NuPAGE 4-12\% Protein Gels (Thermo Fisher Scientific), transferred to PVDF membranes by using the iBlot Gel Transfer Device (Thermo Fisher Scientific), and probed with the appropriate primary antibodies. Membrane-bound primary antibodies were detected using secondary antibodies conjugated with horseradish peroxidase. IBs were developed on films using the enhanced chemiluminescence technique.

\section{Metabolic activity analysis}

Glycolytic activity was determined by measuring the detritiation of $\left[5^{-3} \mathrm{H}\right]$ glucose $e^{56}$. In brief, one million cells were suspended in $0.5 \mathrm{ml}$ fresh media. The experiment was initiated by adding $1 \mu \mathrm{ci}\left[5-{ }^{3} \mathrm{H}\right]$ glucose, and $2 \mathrm{~h}$ later, media were transferred to a $1.5 \mathrm{ml}$ microcentrifuge tube containing $50 \mu \mathrm{l}$ of $5 \mathrm{~N} \mathrm{HCl}$. The microcentrifuge tubes were then placed in $20 \mathrm{ml}$ scintillation vials containing 0.5 $\mathrm{ml}$ water with the vials capped and sealed. ${ }^{3} \mathrm{H}_{2} \mathrm{O}$ was separated from unmetabolized $\left[5-{ }^{3} \mathrm{H}\right]$ glucose by evaporation diffusion for $24 \mathrm{~h}$ at room temperature. A cellfree sample containing $1 \mu \mathrm{ci}\left[5-{ }^{3} \mathrm{H}\right]$ glucose was included as a background control. Glutaminolysis was determined by the rate of ${ }^{14} \mathrm{CO}_{2}$ released from $\left[\mathrm{U}_{-}{ }^{14} \mathrm{C}\right]$ glutamine ${ }^{57}$. In brief, one million cells were suspended in $0.5 \mathrm{ml}$ fresh media. To facilitate the collection of ${ }^{14} \mathrm{CO}_{2}$, cells were dispensed into $7 \mathrm{ml}$ glass vials (TS-13028, Thermo) with a PCR tube containing $50 \mu \mathrm{l}$ of $0.2 \mathrm{M} \mathrm{KOH}$ glued on the sidewall. After adding $0.5 \mu \mathrm{ci}\left[\mathrm{U}_{-}{ }^{14} \mathrm{C}\right.$ ]glutamine, the vials were capped using a screw cap with rubber septum (TS12713, Thermo). The assay was stopped $2 \mathrm{~h}$ later by injection of $100 \mu \mathrm{l}$ of $5 \mathrm{~N} \mathrm{HCl}$ and the vials were kept at room temperate overnight to trap the ${ }^{14} \mathrm{CO}_{2}$. The $50 \mu \mathrm{l}$ of $\mathrm{KOH}$ in the PCR tube was then transferred to scintillation vials containing $10 \mathrm{ml}$ scintillation solution for counting. A cell-free sample containing $0.5 \mu \mathrm{ci}\left[\mathrm{U}-{ }^{14} \mathrm{C}\right]$ glutamine was included as a background control.

\section{Transcriptome analysis}

NB-1643 cells were incubated with $100 \mu \mathrm{M}$ DMF and harvested at designated time points for RNA extraction. RNA samples were then performed microarray by St. Jude Children's Research Hospital. Microarray data were submitted with assigned GEO number as GSE98241.

\section{Chromatin immunoprecipitation-polymerase chain reaction}

ChIP-PCR was done as previously prescribed $^{58}$, using Magna EZ-ChIP (Millipore) according to the manufacturer's protocol. Briefly, after 24-h induction of MYCN expression, the inducible MycN3 cells (a gift of Jason Shohet, Houston, TX, USA $)^{20}$ were cross-linked with $3 \%$ paraformaldehyde for $10 \mathrm{~min}$ and quenched for $5 \mathrm{~min}$ with $125 \mathrm{mmol} / \mathrm{l}$ of glycine. After sonification, cell lysates were spun down and $100 \mu \mathrm{l}$ of supernatant was diluted to $500 \mu \mathrm{l}$ for immunoprecipitation with $5 \mu \mathrm{g}$ of MYCN antibody (Santa Cruz). After serial washing, DNA-protein cross-links were reversed and DNA was extracted for PCR. The PCR primers were designed according to the MYCN ChIP-seq data (GSE94782) in MYCN-amplified NBL cells including NB-1643. Primer sequences are listed in Table S3.

\section{ROS assay}

ROS levels were detected using the $\mathrm{CM}-\mathrm{H}_{2}$ DCFDA probe purchased from Thermo Fisher Scientific. Cells were incubated with $5 \mu \mathrm{M}$ CM-H2DCFDA for $30 \mathrm{~min}$ in serum-free media at $37^{\circ} \mathrm{C}$ and analyzed by flow cytometry immediately.

\section{Animal studies}

C57BL/6NHsd mice were purchased from Envigo. All mice were kept in specific pathogen-free conditions within the research institute at Nationwide Children's Hospital. Animal protocols were approved by the Institutional Animal Care and Use Committee of the research institute at Nationwide Children's Hospital. NB975A2 cells mixed with 70\% Matrigel were injected subcutaneously to mice for the establishment of NBL xenograft model. Mice were randomized into different groups when tumors were 100 to $200 \mathrm{~mm}^{3}$. For the control group, a vehicle control ( $0.8 \%$ hydroxyethyl cellulose) was given by oral gavage every day. For the treated group, DMF was administered orally at a dose of $300 \mathrm{mg} / \mathrm{kg}$ every day till the endpoint of $2,000 \mathrm{~mm}^{3}$. Tumor volumes were determined with the method of tumor length times tumor width times tumor width times 0.52 .

\section{Ethics statement}

All animal experiments were conducted in accordance with institutional animal care and use committee of the Research Institute at Nationwide Children's Hospital approved protocols, designed to minimize the numbers of mice used and to minimize any pain or distress. The named institutional review board or ethics committee specifically approved this study.

\section{Statistical analysis}

$P$ values were calculated with Student's $t$ test. $P$ values $<0.05$ were considered significant, with Pvalues $<0.05$, $<0.01$, and $<0.001$ indicated as $*$, $*$, and $\% *$, respectively. 


\section{Acknowledgements}

We thank Dr. Timothy Cripe, Dr. Pin Yi Wang, Dr. Peter Houghton, and Doris Phelps for valuable discussions and technical assistance. We thank Dr. Rimas Orentas, Dr. Jason Shohet, Dr. Hubert Schorle, and Dr. Chris Lauo for providing valuable reagents to this study. This work was supported by the Davis Bremer Research Pilot Award from The Ohio State University Center for Clinical and Translational Science (to N.S.), R21Al117547 and 1R01Al114581 from National Institute of Health, V2014-001 from the V-Foundation, 128436-RSG-15-180-01LIB from the American Cancer Society and a research grant from CancerFree KIDs Foundation (to R.W.), American Cancer Society-Research Scholar (to J.Y., 130421-RSG-17-071-01-TBG), the National Cancer Institute (to J.Y., R03 CA212802-01A1), the Assisi Foundation of Memphis, the American Lebanese Syrian Associated Charities (ALSAC), the US Public Health Service Childhood Solid Tumor Program Project Grant No. CA23099, and the Cancer Center Support Grant No. 21766 from the National Cancer Institute.

\section{Author details}

${ }^{1}$ Center for Childhood Cancer and Blood Diseases, Hematology/Oncology and BMT, The Research Institute at Nationwide Children's Hospital, The Ohio State University, Columbus, OH, USA. 'Department of Surgery, St. Jude Children's Research Hospital, Memphis, TN 38105 USA

\section{Conflict of interest}

The authors declare that they have no conflict of interest.

\section{Publisher's note}

Springer Nature remains neutral with regard to jurisdictional claims in published maps and institutional affiliations.

Supplementary Information accompanies this paper at (https://doi.org/ 10.1038/541419-018-0295-5).

Received: 10 October 2017 Revised: 1 January 2018 Accepted: 4 January 2018

Published online: 14 February 2018

\section{References}

1. Warburg, O. On the origin of cancer cells. Science 123, 309-314 (1956).

2. DeBerardinis, R. J., Lum, J. J., Hatzivassiliou, G. \& Thompson, C. B. The biology of cancer: metabolic reprogramming fuels cell growth and proliferation. Cell Metab. 7, 11-20 (2008)

3. Vander Heiden, M. G., Cantley, L. C. \& Thompson, C. B. Understanding the Warburg effect: the metabolic requirements of cell proliferation. Science $\mathbf{3 2 4}$ 1029-1033 (2009).

4. Dang, C. V. MYC, metabolism, cell growth, and tumorigenesis. Cold Spring Harbor Perspect. Med. 3 (2013). pii: a014217. doi: 10.1101/cshperspect.a014217.

5. Wang, T., Liu, G. \& Wang, R. The intercellular metabolic interplay between tumor and immune cells. Front. Immunol. 5, 358 (2014).

6. Semenza, G. L. HIF-1: upstream and downstream of cancer metabolism. Curr. Opin. Genet. Dev. 20, 51 (2010).

7. Felsher, D. W. Oncogene addiction versus oncogene amnesia: perhaps more than just a bad habit? Cancer Res. 68, 3081-3086 (2008). discussion 3086.

8. Maris, J. M. Recent advances in neuroblastoma. N. Eng. J. Med. 362, 2202-2211 (2010).

9. Cheung, N. K. \& Dyer, M. A. Neuroblastoma: developmental biology, cancer genomics and immunotherapy. Nat. Rev. Cancer 13, 397-411 (2013).

10. Bown, N. Neuroblastoma tumour genetics: clinical and biological aspects. J. Clin. Pathol. 54, 897-910 (2001)

11. Cohn, S. L. et al. Thelnternational Neuroblastoma Risk Group (INRG) classification system: an INRG Task Force report. J. Clin. Oncol. 27, 289-297 (2009).

12. Qing, G. et al. Combinatorial regulation of neuroblastoma tumor progression by N-Myc and hypoxia inducible factor HIF-1alpha. Cancer Res. 70, 10351-10361 (2010).

13. Xiao, D. et al. Myc promotes glutaminolysis in human neuroblastoma through direct activation of glutaminase 2. Oncotarget 6, 40655-40666 (2015).

14. Qing, G. et al. ATF4 regulates MYC-mediated neuroblastoma cell death upon glutamine deprivation. Cancer Cell 22, 631-644 (2012).
15. Ren, P. et al. ATF4 and N-Myc coordinate glutamine metabolism in MYCNamplified neuroblastoma cells through ASCT2 activation. J. Pathol. 235 90-100 (2015).

16. Altman, B. J., Stine, Z. E. \& Dang, C. V. From Krebs to clinic: glutamine metabolism to cancer therapy. Nat. Rev. Cancer 16, 619-634 (2016).

17. Hay, N. Reprogramming glucose metabolism in cancer: can it be exploited for cancer therapy? Nat. Rev. Cancer 16, 635-649 (2016).

18. Maurer, J. et al. Establishment and controlled differentiation of neural crest stem cell lines using conditional transgenesis. Differentiation 75, 580-591 (2007).

19. Schulte, J. H. et al. MYCN and ALKF1174L are sufficient to drive neuroblastoma development from neural crest progenitor cells. Oncogene 32, 1059-1065 (2013).

20. Slack, A. et al. The p53 regulatory gene MDM2 is a direct transcriptional target of MYCN in neuroblastoma. Proc. Natl. Acad. Sci. USA 102, 731-736 (2005).

21. Sabharwal, S. S. \& Schumacker, P. T. Mitochondrial ROS in cancer: initiators, amplifiers or an Achilles' heel? Nat. Rev. Cancer 14, 709-721 (2014).

22. Sullivan, L. B. \& Chandel, N. S. Mitochondrial reactive oxygen species and cancer. Cancer Metab. 2, 17 (2014).

23. Sullivan, L. B. et al. The proto-oncometabolite fumarate binds glutathione to amplify ROS-dependent signaling. Mol. Cell 51, 236-248 (2013).

24. Zheng, $L$. et al. Fumarate induces redox-dependent senescence by modifying glutathione metabolism. Nat. Commun. 6, 6001 (2015).

25. Duffy, S., So, A. \& Murphy, T. H. Activation of endogenous antioxidant defenses in neuronal cells prevents free radical-mediated damage. J. Neurochem. 71, 69-77 (1998).

26. Gill, A. J. \& Kolson, D. L. Dimethyl fumarate modulation of immune and antioxidant responses: application to HIV therapy. Crit. Rev. Immunol. 33, 307-359 (2013).

27. Kroesen, M. et al. A transplantable TH-MYCN transgenic tumor model in C57B//6 mice for preclinical immunological studies in neuroblastoma. Int. J. Cancer 134, 1335-1345 (2014).

28. Menegon, S., Columbano, A. \& Giordano, S. The dual roles of NRF2 in cancer. Trends Mol. Med. 22, 578-593 (2016).

29. Adam, J. et al. Renal cyst formation in Fh1-deficient mice is independent of the Hif/Phd pathway: roles for fumarate in KEAP1 succination and Nrf2 signaling. Cancer Cell 20, 524-537 (2011).

30. Marshall, G. M. et al. The prenatal origins of cancer. Nat. Rev. Cancer $\mathbf{1 4}$ 277-289 (2014).

31. Dang, C. V. \& Semenza, G. L. Oncogenic alterations of metabolism. Trends Biochem. Sci. 24, 68-72 (1999).

32. Gordan, J. D., Thompson, C. B. \& Simon, M. C. HIF and c-Myc: sibling rivals for control of cancer cell metabolism and proliferation. Cancer Cell 12, 108-113 (2007).

33. Wang, R. et al. The transcription factor Myc controls metabolic reprogramming upon T lymphocyte activation. Immunity 35, 871-882 (2011).

34. Sawai, S., Shimono, A., Hanaoka, K. \& Kondoh, H. Embryonic lethality resulting from disruption of both $\mathrm{N}$-myc alleles in mouse zygotes. New Biol. 3, 861-869 (1991).

35. Knoepfler, P. S., Cheng, P. F. \& Eisenman, R. N. N-myc is essential during neurogenesis for the rapid expansion of progenitor cell populations and the inhibition of neuronal differentiation. Genes Dev. 16, 2699-2712 (2002).

36. Sciacovelli, M., Gaude, E., Hilvo, M. \& Frezza, C. The metabolic alterations of cancer cells. Methods Enzymol. 542, 1-23 (2014).

37. Weinberg, S. E. \& Chandel, N. S. Targeting mitochondria metabolism for cancer therapy. Nat. Chem. Biol. 11, 9-15 (2015).

38. Galluzzi, L., Kepp, O., Vander Heiden, M. G. \& Kroemer, G. Metabolic targets for cancer therapy. Nat. Rev. Drug. Discov. 12, 829-846 (2013).

39. Posternak V., Cole M. D. Strategically targeting MYC in cancer. F1000Research $\mathbf{5}$, 408 (2016) (doi: 10.12688/f1000research.7879.1)

40. Stine, Z. E., Walton, Z. E., Altman, B. J., Hsieh, A. L. \& Dang, C. V. MYC, metabolism, and cancer. Cancer Discov. 5, 1024-1039 (2015).

41. Hogarty, M. D. et al. ODC1 is a critical determinant of MYCN oncogenesis and a therapeutic target in neuroblastoma. Cancer Res. 68, 9735-9745 (2008).

42. Gamble, L. D. et al. Polyamine pathway inhibition as a novel therapeutic approach to treating neuroblastoma. Front. Oncol. 2, 162 (2012).

43. Bassiri, H. et al. Translational development of difluoromethylornithine (DFMO) for the treatment of neuroblastoma. Transl. Pediatr. 4, 226-238 (2015).

44. Evageliou, N. F. et al. Polyamine antagonist therapies inhibit neuroblastoma initiation and progression. Clin. Cancer Res. 22, 4391-4404 (2016). 
45. Trachootham, D. et al. Selective killing of oncogenically transformed cells through a ROS-mediated mechanism by beta-phenylethyl isothiocyanate. Cancer Cell 10, 241-252 (2006).

46. Shaw, A. T. et al. Selective killing of K-ras mutant cancer cells by small molecule inducers of oxidative stress. Proc. Natl. Acad. Sci. USA 108, 8773-8778 (2011).

47. Harris, I. S. et al. Glutathione and thioredoxin antioxidant pathways synergize to drive cancer initiation and progression. Cancer Cell 27, 211-222 (2015).

48. Nicolay, J. P. et al. Dimethyl fumarate restores apoptosis sensitivity and inhibits tumor growth and metastasis in CTCL by targeting NF-kappaB. Blood $\mathbf{1 2 8}$ 805-815 (2016).

49. Sciacovelli, M. et al. Fumarate is an epigenetic modifier that elicits epithelial-tomesenchymal transition. Nature 537, 544-547 (2016).

50. Mrowietz, U. et al. Treatment of psoriasis with fumaric acid esters (Fumaderm). J. Ger. Soc. Dermatol. 5, 716-717 (2007).

51. Walker, F. et al. Fumaderm(R) in daily practice for psoriasis: dosing, efficacy and quality of life. Br. J. Dermatol. 171, 1197-1205 (2014).
52. Fox, R. J. et al. Placebo-controlled phase 3 study of oral BG-12 or glatiramer in multiple sclerosis. N. Eng. J. Med. 367, 1087-1097 (2012).

53. Gold, R. et al. Placebo-controlled phase 3 study of oral BG-12 for relapsing multiple sclerosis. N. Eng. J. Med. 367, 1098-1107 (2012).

54. Shu, L. \& Houghton, P. J. The mTORC2 complex regulates terminal differentiation of C2C12 myoblasts. Mol. Cell. Biol. 29, 4691-4700 (2009).

55. Spandidos, A., Wang, X., Wang, H. \& Seed, B. PrimerBank: a resource of human and mouse PCR primer pairs for gene expression detection and quantification. Nucleic Acids Res. 38, D792-D799 (2010).

56. Hue, L., Sobrino, F. \& Bosca, L. Difference in glucose sensitivity of liver glycolysis and glycogen synthesis. Relationship between lactate production and fructose 2,6-bisphosphate concentration. Biochem. J. 224, 779-786 (1984).

57. Brand, K., Williams, J. F. \& Weidemann, M. J. Glucose and glutamine metabolism in rat thymocytes. Biochem. J. 221, 471-475 (1984).

58. Yang, J. et al. The role of histone demethylase KDM4B in Myc signaling in neuroblastoma. J. Natl. Cancer Inst. 107, djv080 (2015). 